\title{
Bioinformatic gene analysis for potential biomarkers and therapeutic targets of kidney calculi-related renal cell carcinoma
}

Jun Lu

Tongji University School of Medicine

Qunlong Liu

Shanghai Tenth People's Hospital

Huan Liu

Shanghai Tenth People's Hospital

Yunfei Xu ( $\nabla$ xuyunfeibb@sina.com)

Shanghai Tenth People's Hospital

Research article

Keywords: Bioinformatics analysis,Biomarkers,Gene analysis, Kidney calculi, Renal cell carcinoma

Posted Date: April 7th, 2020

DOI: https://doi.org/10.21203/rs.3.rs-20610/v1

License: (9) This work is licensed under a Creative Commons Attribution 4.0 International License. Read Full License 


\section{Abstract}

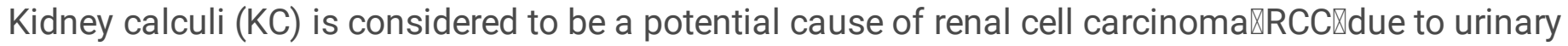
retention, hydronephrosis, pyelonephritis, and carcinoma of renal pelvis. We searched co-expressed genes in order to explore the relationships between kidney calculi $(\mathrm{KC})$ and renal cell carcinoma $\triangle \mathrm{RCC} \triangle$ and reveal potential biomarkers and therapeutic targets of kidney calculi-related renal cell carcinoma.KCrelated differentially expressed genes (DEGs) were identified via bioinformatic analysis Gene Expression Omnibus (GEO) datasets GSE73680 and GSE117518. Simultaneously, RCC-related DEGs were also identified via bioinformatic analysis GEO datasets GSE14994 and GSE40435. Subsequently, co-DEGs of KC-related RCC were found, and extensive target prediction and network analyses methods were used to assess protein-protein interaction (PPI) networks, Gene Ontology (GO) terms and pathway enrichment for DEGs, and co-expressed DEGs coupled with corresponding predicted miRNAs involved in KC and RCC were assessed as well.We identified 832 DEGs in KC and RCC samples. The co-DEGs of VIM $\otimes D C N \otimes W N K 1$ and PXDN coupled with corresponding predicted miRNAs, especially miR-181c-5p and miR-181d-5p may

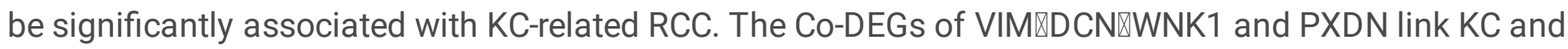
RCC. Finally, the top 5 miRNAs for each Co-DEGs may be potential signaling pathways for KC-relate RCC, especially miR-181c-5p and miR-181d-5p. Therefore, there is an association between KC and RCC and expression of VIM $\triangle D C N \otimes W N K 1$ and PXDN genes may favor KC-related RCC.

\section{Introduction}

Kidney calculi $(\mathrm{KC})$ is one of the most common urological condition. Data from National Health and Nutrition Examination Survey(NHANES) reported in 2012 noted the KC prevalence of $10.6 \%$ in men and $7.1 \%$ among women. ${ }^{1}$ Globally, the incidence and prevalence of $\mathrm{KC}$ have increased over the years. ${ }^{1-3}$ Despite technological advances in the surgical management of $\mathrm{KC}$ have significantly reduced patient morbidity and recovery time, new stone formation and recurrence remain significant health issues. Recurrence rate after the first appearance of symptomatic stones is reported to be $30-50 \%$ within 10 years ${ }^{1}$.Therefore, the research on the mechanism of the occurrence and development of $\mathrm{KC}$ emerges one after another.

Similar to the incidence of kidney stones, the incidence of renal cell carcinoma (RCC), the most common type of renal parenchymal tumor accounting for $90 \%$ of malignant neoplasms arising in the kidney in adults, still continues to keep growing ${ }^{4,5,8}$. Although the best treatment for RCC is resection ${ }^{6}$, advanced patients can only be treated by chemoradiotherapy and other comprehensive methods ${ }^{7}$. If RCC could be predicted, it can be detected early and removed, which will improve the prognosis.

Although there are many risk factors for kidney cancer, such as smoking, obesity and hypertension ${ }^{8}, \mathrm{a}$ Dutch team concluded that early diagnosis of KC (age < 40) was significantly associated with an increased risk of later diagnosis of kidney tumors $(\text { age } \geq 40)^{9}$. They found $\mathrm{KC}$ was associated with an increased RCC risk (HR:1.39,95\% Cl 1.05-1.84) ${ }^{9}$. In fact, previous studies have assessed the relationship 
between kidney stones and RCC and upper tract urothelial carcinoma (UTUC) $)^{10-12}$. In addition, a metaanalysis, based on eight case-control studies and one retrospective cohort study, found an increased risk of RCC and both ureter and renal pelvis cancer in patients with $\mathrm{KC}^{13}$.

The association between kidney cancer and kidney stones is understandable, given the many risk factors they share,such as obesity, diabetes mellitus and several dietary factors. Since RCC in itself is a multigene-related tumor with extremely complex pathogenesis, we speculated that there may be some genetic association between KC and RCC. In this study, we identified co-expressed differentially expressed genes (co-DEGs) of KC and RCC and elucidated molecular mechanisms and pathology of KC-related DEGs (KC-DEGs) and RCC-related DEGs (RCC-DEGs). Finally, we provide a bioinformatic analysis of DEGs and predicted microRNAs (miRNAs) for KC patients prone to occurring RCC.

\section{Methods}

The datasets(GSE73680 and GSE117518) for KC were downloaded from GEO (http://www.ncbi.nlm.nih.gov/geo/) ${ }^{14}$. Expression profiling arrays of GSE73680 were generated using GPL17077 Agilent-039494 SurePrint G3 Human GE v2 8 × 60K Microarray 039381 (Probe Name version), while expression profiling arrays of GSE117518 were generated using GPL21827 Agilent-079487 Arraystar Human LncRNA microarray V4 (Probe Name version). Then, the GeneSpring GX v12.1 software standardized the quantile method and annotated the corresponding data. The data were screened for differential genes through the limma package of R language. Similarly, the datasets (GSE14994 and GSE40435) for RCC were downloaded from CEO. Expression profiling arrays of GSE14994 were generated using GPL3921 [ht_hg-u133a] Affymetrix HT Human Genome U133A Array, standardized by RMA method. While expression profiling arrays of GSE14994 were generated using GPL10558 Illumina humanht-12 V4.0 expression beadchip was used to obtain the corresponding data by quantile standardization method and annotation. The data were screened for differential genes through the limma package of $\mathrm{R}$ language. The criteria we chose were $p<0.05$, foldchange $>2$ or $<0.5$.

The GO Analysis method annotated the gene function of the differential genes based on the GO database to obtain all functions involved in the gene, and then calculated the significance level ( $p$-value) and misjudgment rate (FDR) of each function by Fisher's precise test and multiple comparison test. KEGG (Kyoto Encyclopedia of Genes and Genomes) is a database that systematically analyzes the relationships, gene functions and genome information of Genes (and their coding products). It is helpful for researchers to study Genes and expression information as a whole network. The integrated metabolic pathways provided by KEGG, including the metabolism of carbohydrates, nucleosides, amino acids, and biodegradation of organic compounds, not only provide all possible metabolic pathways, but also provide a comprehensive overview of the enzymes that catalyze each step. KEGG is a powerful tool for metabolic analysis and network research in vivo. Currently, KEGG Pathway is divided into eight categories, including the overall network, metabolic process, genetic information transmission, environmental information transmission, intracellular biological processes, biological systems, human diseases and drug development. Based on the KEGG database, Pathway analysis used Fisher's accurate test and chi square 
test to analyze the significance of Pathway involving the target gene. The screening criteria for both were $\mathrm{P}<0.05$.

PPI networks of KC- and RCC-DEGs were analyzed using the search tool for the retrieval of interacting genes (STRING database, V11; http://string-db.org/) that predicted protein functional associations and protein-protein interactions. Subsequently, Cytoscape software (V3.5.1; http://cytoscape.org/) was applied to visualize and analyze biological networks and node degrees, after downloading analytic results of the STRING database with a combined_score $\geq 0.9$.

Finally, the CTD database was used to find the genes related to RCC or KC. Genes are scored indirectly through a database. Then we chose the top four scores. In addition, we applied online prediction tools utilizing microRNA Data Integration Portal (mirDIP) (http://ophid.utoronto.ca/mirDIP) ${ }^{15}$, and DIANA Tools (http://diana.imis.athena-innovation.gr/DianaTools/) ${ }^{16}$, to predict potential microRNA targeting. Subsequently, we used the mirDIP and DIANA Tools software to predict which of the selected miRNAs could target co-DEGs. We determined 5 top candidate miRNAs based on higher predicted.

\section{Results}

\section{Identification of DEGs}

We identified 15015 probes in GSE73680, of these, 10,468 probes were raised and 4,547 were lowered(Figure1a). Similarly, we identified 2105 probes in GSE117518, of these, 936 probes were raised and 1169 were lowered(Figure1b). The difference genes of the two data sets were intersected, and 343 intersection genes were obtained(Figure1c). In GSE14994, we identified 2332 probes, and 1430 probes were raised and 902 were lowered. While in GSE40435,1764 probes were identified, including 922 upregulated probes and 842 down-regulated probes(Figure1d).The difference genes of the two data sets were intersected, and 832 intersection genes were obtained(Figure1e). The difference genes of the two data sets were intersected, and 832 intersection genes were obtained(Figure1f).

\section{Function enrichment in GO database}

According to the analysis results, the significant function of differentially expressed genes, the number of genes contained and the enrichment degree in the database, the target graph of significance function can be made including biological process, molecular function and cellular component. Figure2a illustrates the significant function of KC-DEGs, and Heatmaps of KC-DEGs in relation to interleukin- 6 secretion, negative regulation of chemokine production were conducted for genes expression and these data appear in Figure2b. Simultaneously, Figure2c and Figure2d has shown the genes expression value in relation to renal system development, small molecule catabolic process above the RCC-DEGs.

\section{KEGG Pathway analysis}


KEGG pathway analysis data appear in Figure3a-b. The results suggesting that the KC-DEGs were mainly enriched in pathways of AMPK signaling pathway (p-value: 4.32E-03), Neurotrophin signaling pathway ( $p$ value: 1.59E-02), TGF-beta signaling pathway ( $p$-value: 2.20E-02), C-type lectin receptor signaling pathway (p-value: 3.22E-02) and Adipocytokine signaling pathway(p-value: 2.98E-02). However, KEGG terms included Phagosome ( $p$-value: 2.68E-13), Valine, leucine and isoleucine degradation ( $p$-value: 2.84E-13), Carbon metabolism ( $p$-value: 1.18E-08), Complement and coagulation cascades ( $p$-value: 2.39E-08) and Glycolysis / Gluconeogenesis (p-value: 3.82E-08) were enriched in RCC-DEGs.

\section{PPI network analysis}

We identified 358 and 445 nodes from PPI network of KC- DEGs and RCC-DEGs, respectively and these data appear in Figure3c-d. Here, the hub nodes, including proteasome 26S subunit,non-ATPase 2 (PSMD2; degree $=16)$, proteasome 20 S subunit alpha $8($ PSMA8; degree $=13)$, STIP1 homology and U-box containing protein $1(S T U B 1$; degree $=13)$, cell division cycle $23(C D C 23$; degree $=12)$, integrin linked kinase $(I L K$; degree $=12)$ are considering as hub-genes in related to KC maintaining. However, the hubgenes, involved in kininogen $1(\mathrm{KNG} 1$; degree $=56)$, complement $\mathrm{C} 3(\mathrm{C} 3$; degree $=51)$, quiescin sulfhydryl oxidase $1(\mathrm{QSOX} 1$; degree $=44)$, apolipoprotein $\mathrm{B}(A P O B$; degree $=42)$, and $\mathrm{fCG}(\mathrm{FCG}$; degree $=40)$ are demonstrated in RCC-DEGs with a relative higher degree.

\section{Identification of functional and pathway enrichment among predicted miRNAs and Co-DEGs}

The CTD database showed that Co-DEGs targeted KC and RCC. According to databases score genes indirectly, the inclusion criteria is score $>10$. Thus, four genes were selected-Vimentin(VIM) $\otimes$ Decorin(DCN) $₫$ WNK lysine deficient protein kinase 1(WNK1) खperoxidasin(PXDN). The Figure4 showed the relationship between genes and diseases. In renal carcinoma, the disease most associated with DCN is RCC, while in calculi, the disease is KC(Figure4a). In addition, the same condition happens in VIM, WNK1 and PXDN(Figure4b-d). Prediction analysis using mirDIP and DIANA bioinformatic tools identified the top 5 selected miRNAs targeting each Co-DEG involved in KC-related RCC and these data appear in Table1. These data enable us to understand how predicted miRNAs are related to KC-related RCC progress.

\section{Discussion}

With the high incidence of $\mathrm{KC}$, its consequent harm should also be highly concerned, which also including the potential of progressing to be RCC. In clinical practice, early and comprehensive screening for KC patients is essential to prevent the occurrence of RCC. However the etiology of RCC is not clear, it is widely believed that RCC is closely related to KC, obesity, and hypertension. Although the concurrence of $\mathrm{KC}$ and RCC may be influenced by a series of confounding factors, there is still a close relationship between these two disease. Previous studies are suggested that $\mathrm{KC}$ can be used as a risk factor for postoperative recurrence in KC patients with RCC. Jeroen's group ${ }^{9}$ had reported that nearly half of all RCC cases in their study could be attributed to $\mathrm{KC}$ based on the population attributable fraction using a multivariable-adjusted HR of 3.08. Facing with such a high risk, the process of its occurrence and 
development is worth exploring. KC patients usually have hydronephrosis, which can increase the risk of nephropathy and the disease process, and significantly affect the prognosis of disease. But at present there are few studies focused on the relationship between KC and RCC.

In our study, major Co-DEGs included VIM,DCN匹WNK1 and PXDN, which are associated with both KC and RCC. The VIM bring a high influence score in RCC and KC, especially in RCC. The result is consistent with previous researches considering the invasion and metastasis of RCC via altering the miR490-3p/vimentin signals ${ }^{17}$. VIM plays an important role in a variety of tumors, not only in RCC, but also in such as breast carcinoma $^{18}$, nasopharyngeal carcinoma ${ }^{19,20}$, Glioblastoma ${ }^{21}$ and gastric cancer ${ }^{22}$. In addition, Yu' group found extracellular vimentin modulates human dendritic cell activation, in order to prevent tissue-damage from contributing to the development of autoimmunity ${ }^{23}$. Similarly, Santos et al suggests that VIM may be a key regulator of the NLRP3 inflammasome ${ }^{24}$. Both of them indicated that VIM is closely associated with inflammation. It may be an implication that KC suffers may have a risk of developing RCC via altering the VIM.

As for DCN, in our results, it's mainly associated with kidney neoplasms, RCC, KC, renal colic and chronic kidney disease-mineral and bone disorder. According to Xu's research, they illustrated that the loci of DCN deficiency is significantly associated with RCC growth and metastasis ${ }^{25}$. Similarly, it's also related to inflammation ${ }^{26}$. Indeed, Merline et al considered DCN could control inflammation and cancer ${ }^{27}$.

Additionally, WNK1 could promote renal tumor progression according to Kim's study ${ }^{28}$. Some researchers also deem it's related to immunity and inflammation ${ }^{30}$. More interestingly, PXDN is considered to be associated with renal fibrosis in the murine unilateral ureteral obstruction model ${ }^{29}$.Thus, there may be a relationship between $\mathrm{KC}$ and $\mathrm{RCC}$ that they may arise from loci mutations or gene variants.

Although our study may reveal some potential relationships between $\mathrm{KC}$ and RCC, it can not consider the biological mechanism must relate KC to RCC. In general, previous studies considered that chronic irritation and infection recruit inflammatory cells, which secrete cytokines and chemokines. In turn, free radical species from oxygen and nitrogen are produced, facilitating the onset of cancer through, among others, increasing cell proliferation ${ }^{31-33}$. However, the Co-DEGs in our study are compatible with the supposition.

Finally, the results of KEEG and GO pathway analysis about Co-DEGs illustrate some possible pathway to investigate the effect of KC to RCC. We found that miR-181d-5p, miR-181c-5p were Co-DEGs and may be potential signaling pathway of KC-related RCC. In previous studies, miR-181c-5p had been reported it could promote an inflammatory response leading to diseases progression ${ }^{34-35}$. While miR-181d-5p had been considered to induce inhibition of oxidarive stress response ${ }^{36}$. Obviously, it coincides with the previous supposition again.

\section{Conclusion}


The Co-DEGs of VIM,DCN凹WNK1 and PXDN link KC and RCC. Finally, the top 5 miRNAs for each Co-DEGs may be potential signaling pathways for KC-relate RCC, especially miR-181c-5p and miR-181d-5p. Therefore, there is an association between KC and RCC, and expression of VIM $\square$ DCN $\square W N K 1$ and PXDN genes may favor KC-related RCC.

\section{Limitation}

This study is a microarray analysis that all the results based on gene expression value. Therefore, validation should be carried out both in vitro, in vivo and clinical trials. The genes and potential pathway we found do not appear to be present in previous studies about KC-related RCC. Thus, our study may provide some directions for further research.

\section{Declarations}

\section{Acknowledgments}

The excellent technical assistance of Shanghai Qisheng Biological Preparation Co., Ltd is gratefully acknowledged. We would like to acknowledge the KEGG, GEPIA, TCGA, Oncomine, cBioPortal and starbase databases for free use.

Funding: Funding information is not applicable.

\section{Author contributions:}

Yunfei Xu: Concept/design, critical revision of the article, approval of the article.

Jun Lu: Concept/design, data analysis/interpretation, drafting of the article, approval of the article.

Qunlong Liu:Data collection, data analysis/interpretation, make the figure,approval of the article.

Huan Liu: Concept/design,revision of the article, approval of the article.

\section{Conflict of Interest Statement}

The authors of this manuscript have no conflict of interest.

\section{References}

1.Dion M, Ankawi G, Chew B, Paterson R, Sultan N, Hoddinott P, Razvi H囚CUA guideline on the evaluation and medical management of the kidney stone patient-2016 update. Can Urol Assoc J 10(11-12),2016.

2.Romero V, Akpinar $\mathrm{H}$, Assimos DG:Kidney stones: a global picture of prevalence, incidence, and associated risk factors. Rev Urol 12(2-3):e86-96,2010. 
Sfoungaristos S, Gofrit ON, Yutkin V, Pode D, Duvdevani M: Prevention of renal stone disease recurrence. A systematic review of contemporary pharmaceutical options. Expert Opin Pharmacother 16(8):120918,2015 .

4.Yuexin Xu,Alicia J.Morales,Michael J.Cargill,Andrea M.H.Towlerton,David G.Coffey,Edus H.Warren,Scott S.Tykodi:Preclinical development of T-call receptor-engineered T-cell therapy targeting the 5T4 tumor antigen on renal cell carcinoma.Cancer Immunol Immunother 68(12):1979-1993,2019.

5.Jemal A, Siegel R, Ward E, Hao Y, Xu J, Murray T, Thun MJ: Cancer statistics, 2008. CA Cancer J Clin 58(2):71-96,2008.

Gong D, Zhang J, Chen Y, Xu Y, Ma J, Hu G, Huang Y, Zheng J, Zhai W, Xue W:The m6A-suppressed P2RX6 activation promotes renal cancer cells migration and invasion through ATP-induced Ca2+ influx modulating ERK1/2 phosphorylation and MMP9 signaling pathway. J Exp Clin Cancer Res 38(1):233,2019.

7.Miao D, Margolis CA, Gao W, Voss MH, Li W, Martini DJ, Norton C, Bossé D,Wankowicz SM, et al:Genomic correlates of response to immune checkpoint therapies in clear cell renal cell carcinoma. Science 359(6377):801-806,2018.

Vitale MG, Bracarda S, Cosmai L, Crocetti E, Di Lorenzo G, Lapini A, Mandressi A, Martorana G, Masini C, et al:Management of kidney cancer patients: 2018 guidelines of the Italian Medical Oncology Association (AIOM).Tumori Journal,105(4S) 3-12,2019.

van de Pol JAA, van den Brandt PA, Schouten LJ:Kidney stones and the risk of renal cell carcinoma and upper tract urothelial carcinoma: the Netherlands Cohort Study.Br. J. Cancer 120(3):368-374,2019.

McCredie M, Stewart JH: Risk factors for kidney cancer in New South Wales, Australia. II. Urologic disease, hypertension, obesity, and hormonal factors. Cancer Causes Control 3(4):323-331,1992 .

Chow WH, Lindblad P, Gridley G, Nyrén O, McLaughlin JK, Linet MS, Pennello GA, Adami HO, Fraumeni JF Jr:Risk of urinary tract cancers following kidney or ureter stones. J Natl Cancer Inst 89(19):1453-1457 ,1997.

12.Chung SD, Liu SP, Lin HC: A population-based study on the association between urinary calculi and kidney cancer. Can Urol Assoc J 7(11-12):E716-21, 2013

Cheungpasitporn W, Thongprayoon C, O'Corragain OA, Edmonds PJ, Ungprasert P, Kittanamongkolchai W, Erickson SB:The risk of kidney cancer in patients with kidney stones: a systematic review and metaanalysis. QJM 108(3):205-12,2015.

Barrett T, Wilhite SE, Ledoux P, Evangelista C, Kim IF, Tomashevsky M, Marshall KA, Phillippy KH, Sherman PM, Holko M, et al:NCBI GEO: archive for functional genomics data sets-update. Nucleic Acids Res 41(Database issue):D991-D995,2013. 
15.Pio G, Malerba D, D'Elia D, Ceci M: Integrating microRNA target predictions for the discovery of gene regulatory networks: a semi-supervised ensemble learning approach.BMC Bioinformatics 15(Suppl 1):S4,2014.

16.Paraskevopoulou MD, Georgakilas G, Kostoulas N, Vlachos IS, Vergoulis T, Reczko M, Filippidis C, Dalamagas T, Hatzigeorgiou AG: DIANA-microT web server v.50: service integration into miRNA functional analysis workflows. Nucleic Acids Res. W169-173,2013.

17.Bai J, Yeh S, Qiu X, Hu L, Zeng J, Cai Y, Zuo L, Li G, Yang G, Chang C: TR4 nuclear receptor promotes clear cell renal cell carcinoma (ccRCC) vasculogenic mimicry (VM) formation and metastasis via altering the miR490-3p/vimentin signals. Oncogene. 37(44):5901-5912,2018.

18.Messica Y, Laser-Azogui A, Volberg T, Elisha Y, Lysakovskaia K, Eils R, Gladilin E, Geiger B, Beck R:The role of Vimentin in Regulating Cell Invasive Migration in Dense Cultures of Breast Carcinoma Cells. Naonlett. 17 (11):6941-6948,2017.

19.Wang W, Yi M, Zhang R, Li J, Chen S, Cai J, Zeng Z, Li X, Xiong W et al: Vimentin is a crucial target for anti-metastasis therapy of nasopharyngeal carcinoma.Mol Cell Biochem. 438(1-2):47-57,2018.

20.Lu W, Luo JY, Wu MH, Hou JY, Yang X, Chen G, Feng ZB:Expression of vimentin in nasopharyngeal carcinoma and its possible molecular mechanism:A study based on immunohistochemistry and bioinformatics analysis. Pathol Res Pract. 215(5):1020-1032,2019.

21.Zhao J, Zhang L, Dong X, Liu L, Huo L, Chen H: High Expression of Vimentin is Associated With Progression and a Poor Outcome in Glioblastoma. Appl Immunohistochem Mol Morphol. 26(5):337344,2018 .

22.Yin S, Chen FF, Yang GF:Vimentin immunohistochemical expression as a prognostic factor in gastric cancer: A meta-analysis. Pathol Res Pract. 214(9):1376-1380,2018.

23.Yu MB, Guerra J, Firek A, Langridge WHR: Extracellular vimentin modulates human dendritic cell activation. Mol Immunol. 104:37-46,2018.

24.dos Santos G, Rogel MR, Baker MA, Troken JR, Urich D, Morales-Nebreda L, Sennello JA, Kutuzov MA, Sitikov A: Vimentin regulates activation of the NLRP3 inflammasome. Nat Commun. 6:6574,2015.

25.Xu Y, Xia Q, Rao Q, Shi S, Shi Q, Ma H, Lu Z, Chen H, Zhou X:DCN deficiency promotes renal cell carcinoma growth and metastasis through downregulation of P21 and E-cadherin.Tumour Tumour Biol. 37(4): 5171-83,2016.

26.Bocian C, Urbanowitz AK, Owens RT, lozzo RV, Götte M, Seidler DG: Decorin potentiates interferon-y activity in a model of allergic inflammation. J Biol Chem. 288(18):12699-711,2013. 
27.Merline R, Moreth K, Beckmann J, Nastase MV, Zeng-Brouwers J, Tralhão JG, Lemarchand P, Pfeilschifter J, Schaefer RM, et al: Signaling by the matrix proteoglycan decorin controls inflammation and cancer through PDCD4 and MicroRNA-21. Sci Signal. 4(199):ra75,2011.

28.Kim JH,Hwang KH, Eom M, Kim M, Park EY, Jeong Y, Park KS, Cha SK: WNK1 promotes renal tumor progression by activating TRPC6-NFAT pathway. FASEB J. 33(7):8588-8599,2019.

29.Colon S, Luan H, Liu Y, Meyer C, Gewin L, Bhave G: Peroxidasin and eosinophil peroxidase, but not myeloperoxidase, contribute to renal fibrosis in the murine unilateral ureteral obstruction model. $\mathrm{Am} \mathrm{J}$ Physiol Renal Physiol. 316(2):F360-F371,2019.

30.Köchl R, Thelen F, Vanes L, Brazão TF, Fountain K, Xie J, Huang CL, Lyck R, Stein JV, et al:WNK1 kinase balances T cell adhesion versus migration in vivo. Nat Immunol. 17(9):1075-83,2016.

31.Federico A1, Morgillo F, Tuccillo C, Ciardiello F, Loguercio C: Chronic inflammation and oxidative stress in human carcinogenesis. Int. J. Cancer. 121(11), 2381-2386,2007.

32.Chung SD1, Liu SP, Lin HC: A population-based study on the association between urinary calculi and kidney cancer. Can. Urol. Assoc. 7(11): E716-E721,2013.

33.Cheungpasitporn W, Thongprayoon C, O'Corragain OA, Edmonds PJ, Ungprasert P, Kittanamongkolchai W, Erickson SB: The risk of kidney cancer in patients with kidney stones: a systematic review and meta-analysis. QJM 108(3): 205-212, 2015.

34.Cao DW, Liu MM, Duan R, Tao YF, Zhou JS, Fang WR, Zhu JR, Niu L, Sun JG:The IncRNA Malat1 functions as a ceRNA to contribute to berberine-mediated inhibition of HMGB1 by sponging miR-181c-5p in poststroke inflammation.Acta Pharmacol. Sin. 41(1):22-33,2020.

35.Ji LJ, Li F, Zhao P, Weng LP, Wei J, Yan J, Liu LN:Silencing interleukin 1a underlies a novel inhibitory role of miR-181c-5p in alleviating low-grade inflammation of rats with irritable bowel syndrome. Cell. Biochem. 120(9):15268-15279,2019.

36.Zhang M, Wang X, Yao J, Qiu Z:Long non-coding RNA NEAT1 inhibits oxidative stress-induced vascular endothelial cell injury by activating the miR-181d-5p/CDKN3 axis. Artif Cells Nanomed Biotechnol 47(1):3129-3137,2019.

\section{Table}

TableI The Gene Ontology (GO) terms and Kyoto Encyclopedia of Genes and Genomes (KEGG) pathways enrichment among predicted miRNAs and Co-DEGs 


\begin{tabular}{|c|c|c|c|c|}
\hline$\overline{\text { Genes }}$ & $\begin{array}{l}\text { Predicted } \\
\text { miRNAs }\end{array}$ & Category & Function & P-value \\
\hline \multirow[t]{10}{*}{$\overline{\text { VIM }}$} & $\begin{array}{l}\text { hsa-miR-30e- } \\
5 p\end{array}$ & $\begin{array}{l}\text { KEGG } \\
\text { pathway }\end{array}$ & Axon guidance & $7.71 \mathrm{E}-13$ \\
\hline & $\begin{array}{l}\text { hsa-miR-124- } \\
3 p\end{array}$ & & Long-term depression & $2.47 \mathrm{E}-12$ \\
\hline & hsa-miR-320a & & Amphetamine addiction & 8.13E-09 \\
\hline & hsa-miR-320b & & Gap junction & $1.04 \mathrm{E}-08$ \\
\hline & $\begin{array}{l}\text { hsa-miR-30d- } \\
5 p\end{array}$ & & $\begin{array}{l}\text { Arrhythmogenic right } \\
\text { cardiomyopathy (ARVC) }\end{array}$ & 3.14E-08 \\
\hline & & GO terms & cell-cell signaling & $7.25 \mathrm{E}-06$ \\
\hline & & & cytoskeleton organization & $1.62 \mathrm{E}-05$ \\
\hline & & & immune system process & 3.47E-05 \\
\hline & & & commissural neuron axon guidance & 8.84E-05 \\
\hline & & & $\begin{array}{l}\text { regulation of cellular response to growtl } \\
\text { factor stimulus }\end{array}$ & 0.00035515 \\
\hline \multirow[t]{14}{*}{ DCN } & $\begin{array}{l}\text { hsa-miR-590- } \\
3 p\end{array}$ & $\begin{array}{l}\text { KEGG } \\
\text { pathway }\end{array}$ & Amphetamine addiction & $1.11 \mathrm{E}-16$ \\
\hline & $\begin{array}{l}\text { hsa-miR- } \\
181 d-5 p\end{array}$ & & Phosphatidylinositol signaling system & $1.73 \mathrm{E}-10$ \\
\hline & $\begin{array}{l}\text { hsa-miR- } \\
181 c-5 p\end{array}$ & & Glioma & $6.30 \mathrm{E}-10$ \\
\hline & $\begin{array}{l}\text { hsa-miR- } \\
181 a-5 p\end{array}$ & & Long-term potentiation & 3.19E-05 \\
\hline & $\begin{array}{l}\text { hsa-miR- } \\
181 b-5 p\end{array}$ & & post-translational protein modification & 8.88E-16 \\
\hline & & GO terms & $\begin{array}{l}\text { epidermal growth factor receptor signaling } \\
\text { pathway }\end{array}$ & $3.77 \mathrm{E}-15$ \\
\hline & & & $\begin{array}{l}\text { nucleobase-containing compound cataboli } \\
\text { process }\end{array}$ & c $1.03 E-14$ \\
\hline & & & membrane organization & $4.40 \mathrm{E}-12$ \\
\hline & & & $\begin{array}{l}\text { fibroblast growth factor receptor signaling } \\
\text { pathway }\end{array}$ & $9.96 \mathrm{E}-12$ \\
\hline & & & inositol phosphate metabolic process & $6.04 \mathrm{E}-11$ \\
\hline & & & regulation of nitric-oxide synthase activity & $2.72 \mathrm{E}-10$ \\
\hline & & & nitric oxide metabolic process & $4.92 \mathrm{E}-10$ \\
\hline & & & phosphatidylinositol-mediated signaling & $1.15 E-09$ \\
\hline & & & phospholipid metabolic process & 3.59E-09 \\
\hline \multicolumn{2}{|c|}{$\begin{array}{l}\text { WNK1 hsa-miR-124- } \\
\text { 3p }\end{array}$} & $\begin{array}{l}\text { KEGG } \\
\text { pathway }\end{array}$ & Estrogen signaling pathway & $2.71 \mathrm{E}-11$ \\
\hline
\end{tabular}


hsa-miR-128-

$3 p$

hsa-miR- GO terms phospholipid metabolic process

$181 d-5 p$

hsa-miR-

181c-5p

hsa-miR-454-

$3 p$

\section{$\begin{array}{ll}\text { PXDN hsa-miR-9-5p } & \text { KEGG } \\ \text { pathway }\end{array}$} hsa-miR-29c- GO terms $3 p$

hsa-miR-29a$3 \mathrm{p}$

hsa-miR-29b$3 p$

hsa-miR-202$3 p$
Bacterial invasion of epithelial cells

0.00015868

$2.44 \mathrm{E}-08$

nucleobase-containing compound catabolic 2.77E-08 process

platelet activation

4.72E-08

transcription initiation from RNA 1.55E-07 polymerase II promoter

glycerophospholipid biosynthetic process

1.75E-07

Thyroid hormone signaling pathway

$6.94 \mathrm{E}-08$

response to stress

5.23E-08

cellular protein metabolic process

6.94E-08

nitric oxide metabolic process

1.43E-07

chondroitin sulfate metabolic process

2.06E-06

regulation of nitric-oxide synthase activity

1.41E-05

negative regulation of translation

\section{Figures}



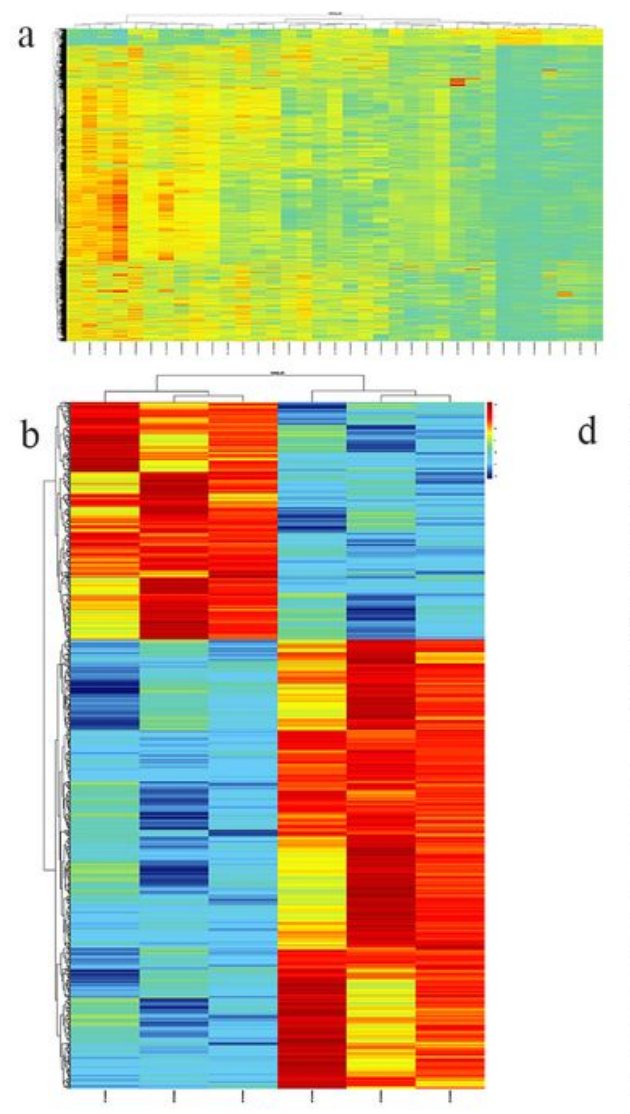

d
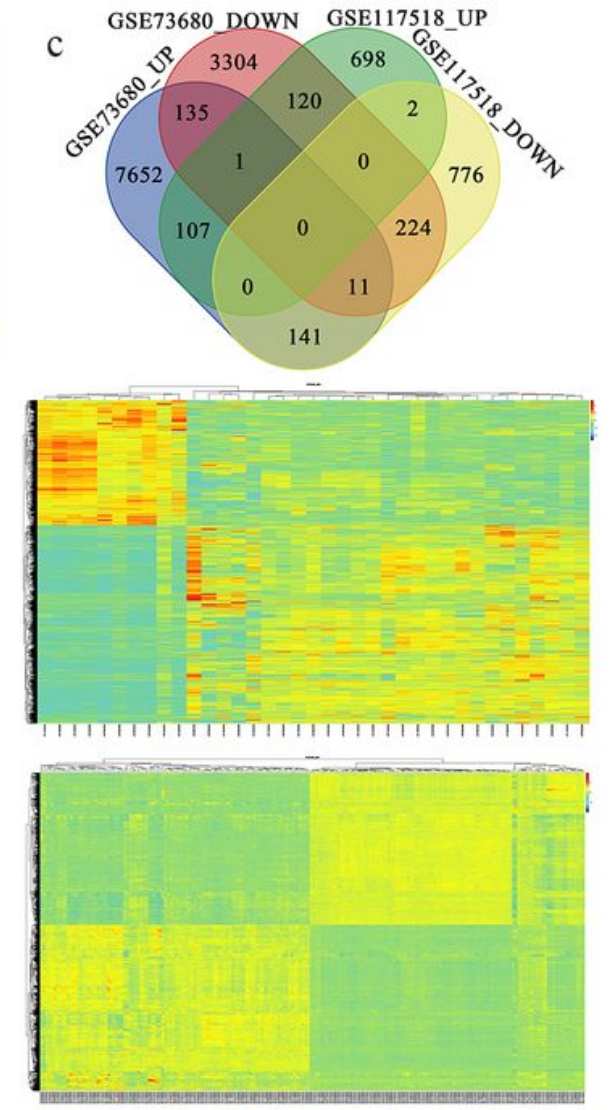

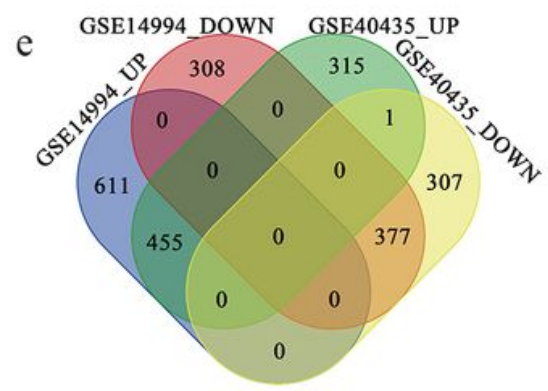

$\mathrm{f}$

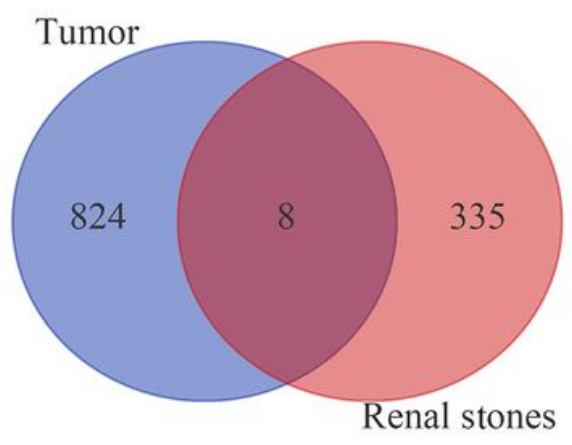

\section{Figure 1}

We identified 15015 probes in GSE73680, of these, 10,468 probes were raised and 4,547 were lowered(Figure1a). Similarly, we identified 2105 probes in GSE117518, of these, 936 probes were raised and 1169 were lowered(Figure1b).The difference genes of the two data sets were intersected, and 343 intersection genes were obtained(Figure1c). In GSE14994, we identified 2332 probes, and 1430 probes were raised and 902 were lowered. While in GSE40435,1764 probes were identified, including 922 upregulated probes and 842 down-regulated probes(Figure1d). The difference genes of the two data sets were intersected, and 832 intersection genes were obtained(Figure1e). The difference genes of the two data sets were intersected, and 832 intersection genes were obtained(Figure1f). 
a

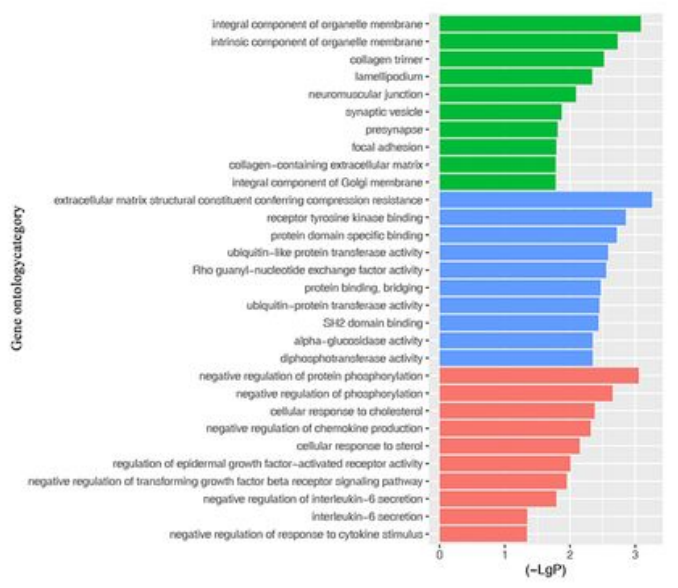

b
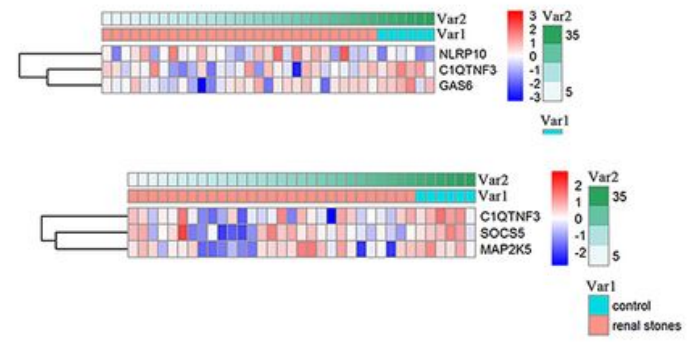

$\mathrm{c}$
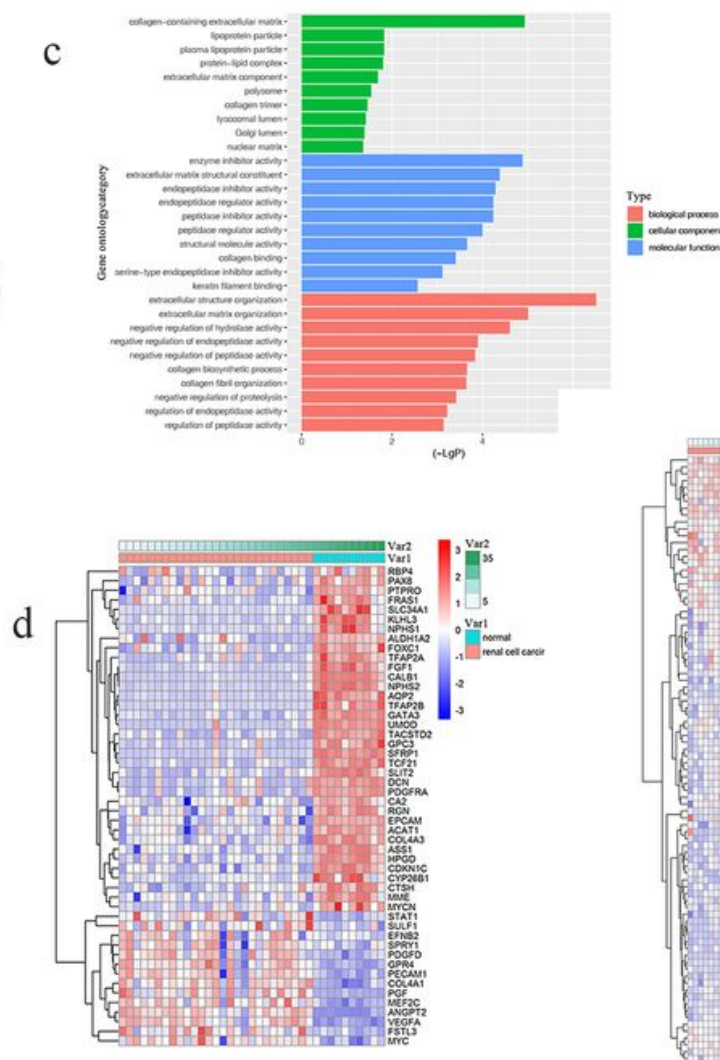

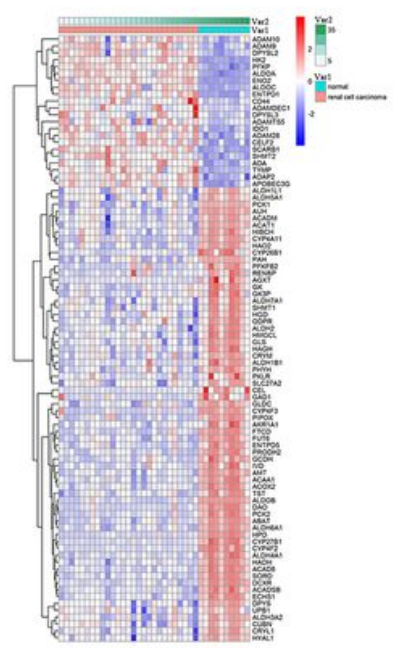

Figure 2

Figure2a illustrates the significant function of KC-DEGs, and Heatmaps of KC-DEGs in relation to interleukin- 6 secretion, negative regulation of chemokine production were conducted for genes expression and these data appear in Figure2b. Simultaneously, Figure2c and Figure2d has shown the genes expression value in relation to renal system development, small molecule catabolic process above the RCC-DEGs. 
a
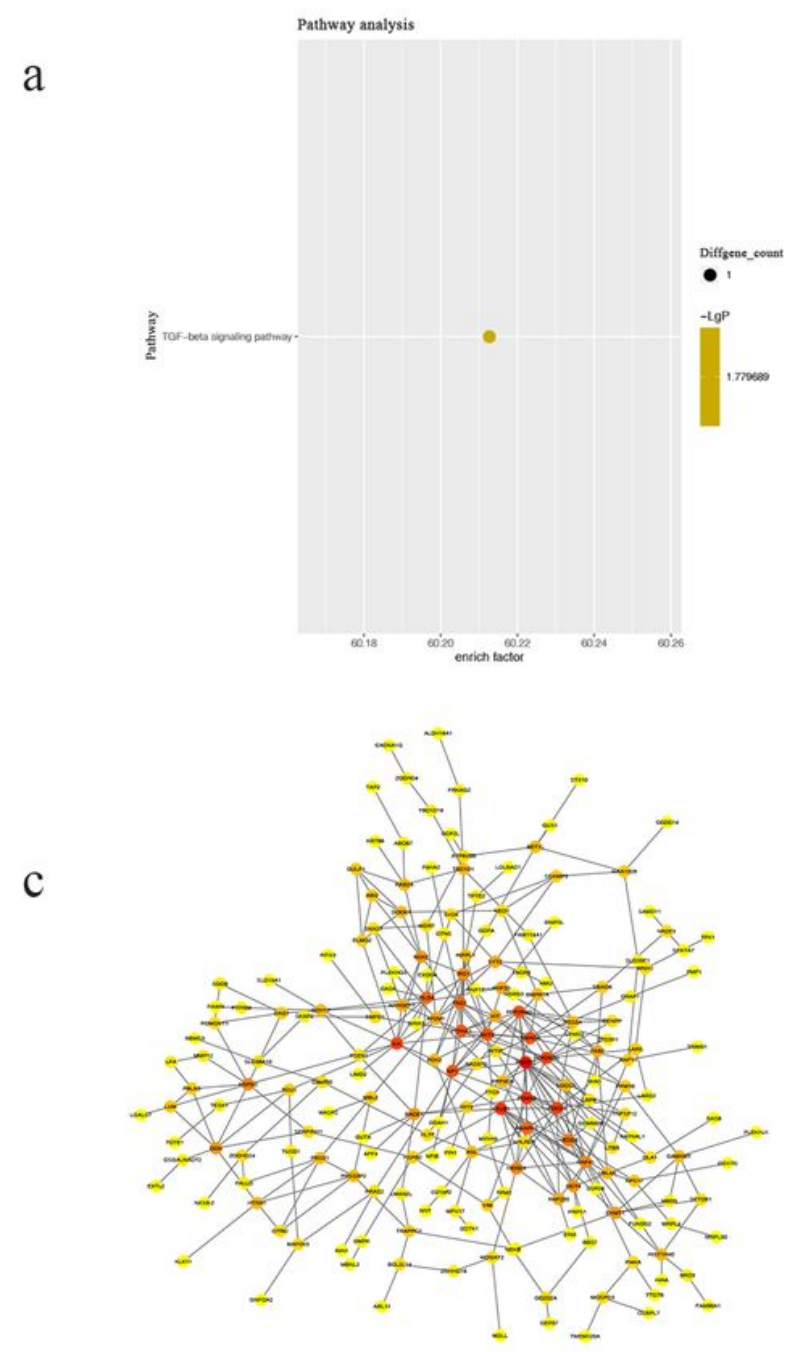

$\mathrm{b}$
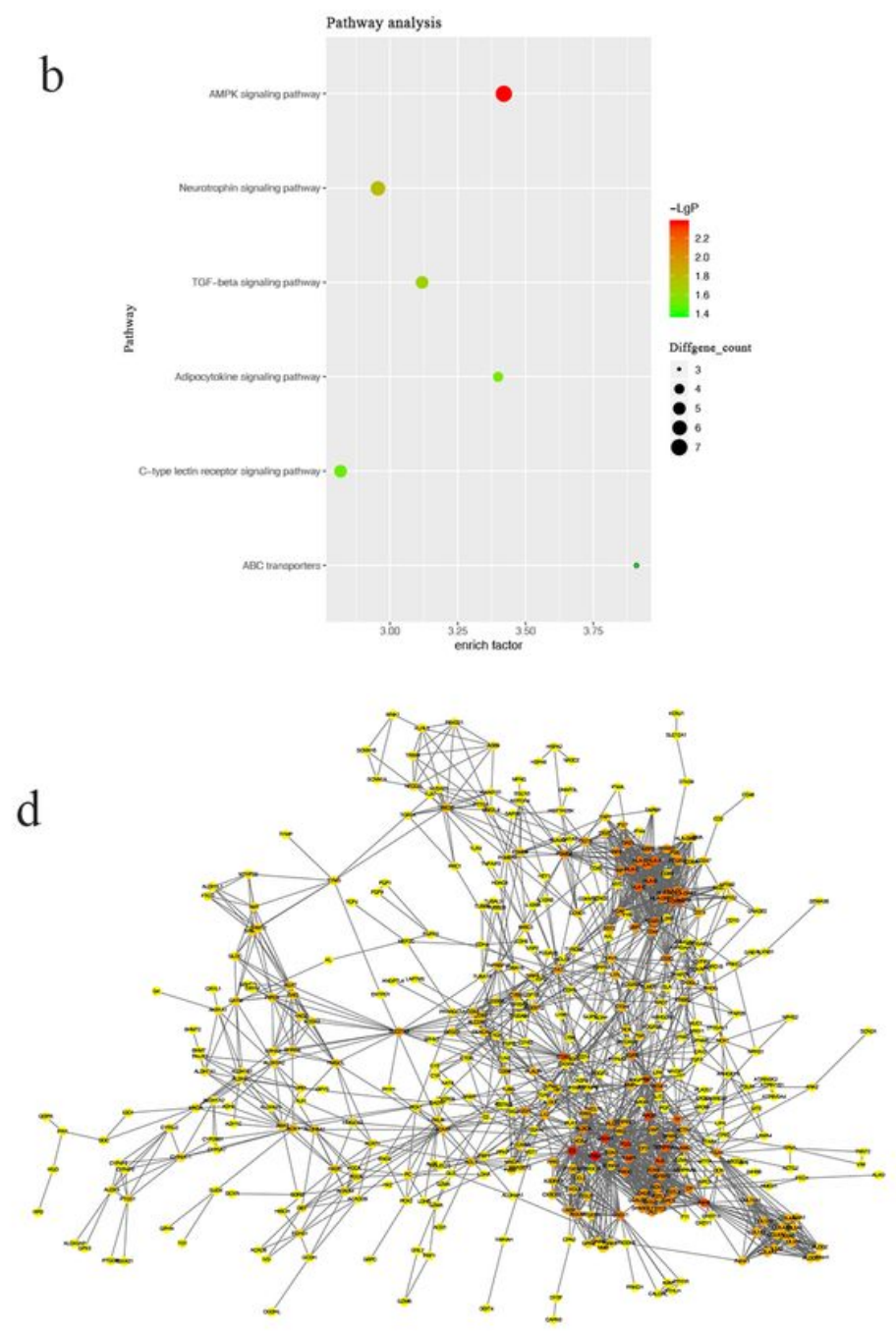

Figure 3

KEGG pathway analysis data appear in Figure3a-b. We identified 358 and 445 nodes from PPI network of KC-DEGs and RCC-DEGs, respectively and these data appear in Figure3c-d. 
a

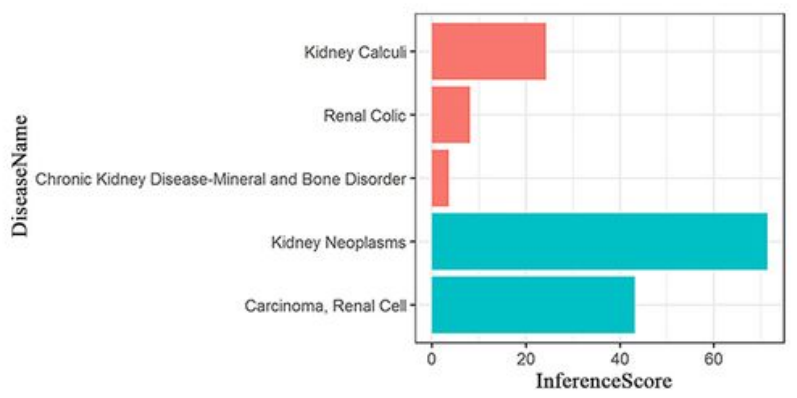

b

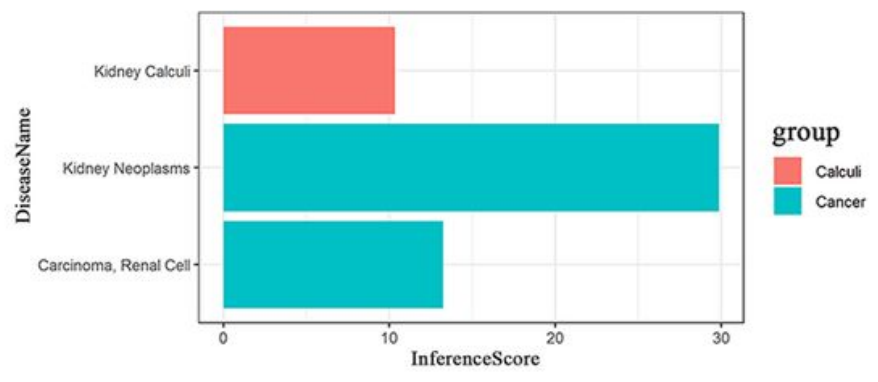

C

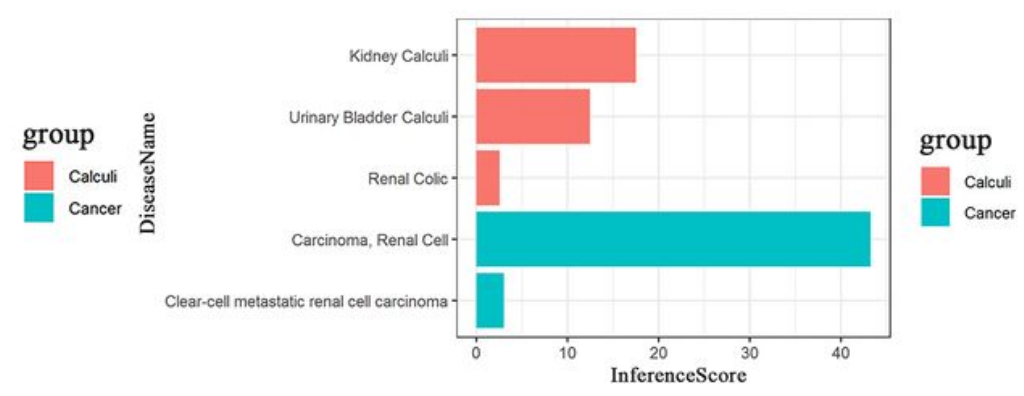

$\mathrm{d}$

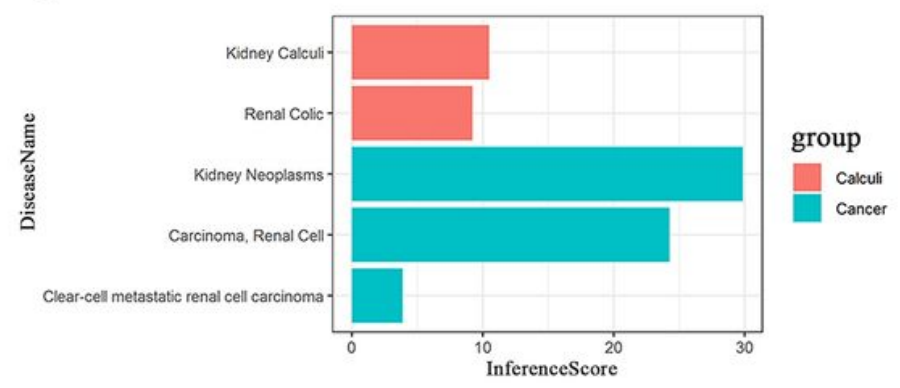

\section{Figure 4}

The Figure4 showed the relationship between genes and diseases. In renal carcinoma, the disease most associated with DCN is RCC, while in calculi, the disease is KC(Figure4a). In addition, the same condition happens in VIM, WNK1 and PXDN(Figure4b-d). 